\title{
Quantum Teleportation Between Discrete and Continuous Encodings of an Optical Qubit
}

\author{
Alexander E. Ulanov, ${ }^{1,2}$ Demid Sychev, ${ }^{1,3}$ Anastasia A. Pushkina, ${ }^{1,4}$ Ilya A. Fedorov, ${ }^{1,5}$ and A. I. Lvovsky ${ }^{1,4,5}$ \\ ${ }^{1}$ International Center for Quantum Optics and Quantum Technologies (Russian Quantum Center), Skolkovo, Moscow 143025, Russia \\ ${ }^{2}$ Moscow Institute of Physics and Technology, 141700 Dolgoprudny, Russia \\ ${ }^{3}$ Moscow State Pedagogical University, E.V. Shpolsky Chair of Theoretical Physics, \\ M. Pirogovskaya Street 29, Moscow 119991, Russia \\ ${ }^{4}$ Institute for Quantum Science and Technology, University of Calgary, Calgary, Alberta T2N 1N4, Canada \\ ${ }^{5}$ P. N. Lebedev Physics Institute, Leninskiy prospect 53, Moscow 119991, Russia
}

(Received 15 July 2016; revised manuscript received 29 November 2016; published 17 April 2017)

\begin{abstract}
The transfer of quantum information between physical systems of a different nature is a central matter in quantum technologies. Particularly challenging is the transfer between discrete and continuous degrees of freedom of various harmonic oscillator systems. Here we implement a protocol for teleporting a continuousvariable optical qubit, encoded by means of low-amplitude coherent states, onto a discrete-variable, singlerail qubit - a superposition of the vacuum and single-photon optical states — via a hybrid entangled resource. We test our protocol on a one-dimensional manifold of the input qubit space and demonstrate the mapping onto the equator of the teleported qubit's Bloch sphere with an average fidelity of $0.83 \pm 0.04$. Our work opens up the way to the wide application of quantum information processing techniques where discrete- and continuous-variable encodings are combined within the same optical circuit.
\end{abstract}

DOI: 10.1103/PhysRevLett.118.160501

Introduction.-The real-world application of quantum technologies in many cases requires the simultaneous use of physical systems of a different nature [1] in a hybrid fashion. For example, one of the most promising platforms for quantum information processing is superconducting circuitry, and the best storage performance is achieved in atomic systems, whereas the transmission of that information in space is better realized with photons.

Within quantum optics, the hybrid paradigm consists in a symbiotic involvement of states and methods from discrete(DV) and continuous- (CV) variable domains. Fortés of both can thereby be employed at once; for example, the continuous part takes advantage of the deterministic state preparation and relatively simple integration with existing information technologies [2], while the discrete side benefits from a natural photodetection basis and "builtin" entanglement distillation after losses [3]. This flexibility allows hybrid quantum technologies to offer protocols which are superior to both pure CV and DV solutions [4]. For example, using hybrid methods in quantum computing [5] and error correction [6] is favorable, compared to the mainstream $\mathrm{CV}$ and $\mathrm{DV}$ protocols, in terms of the number of resources required. The longdistance distribution of $\mathrm{CV}$ entanglement requires hybrid processing to mitigate the effect of propagation losses $[7,8]$.

Taking advantage of these protocols, though, requires a procedure for transferring quantum data between continuous and discrete qubit systems [4,9]; hybrid teleportation is therefore identified as one of the three key priorities for quantum teleportation science [10]. In this work, we make a step towards this goal, demonstrating quantum teleportation [11] by means of a hybrid entangled channel. Specifically, we develop a technique to teleport a CV qubit, encoded as a superposition of coherent states, onto a DV qubit-a superposition of the vacuum and single-photon Fock states. The protocol is conceptually different from the teleportation of photonic bits by a hybrid technique [12], in which both the input and output states are discrete-variable single-rail qubits.

Concept.-The protocol is carried out via an entangled resource state $[6,13]$

$$
\left.\left.|R\rangle_{C D}=K_{-} \mid \text {cat }_{-}\right\rangle_{C}|0\rangle_{D}+K_{+} \mid \text {cat }_{+}\right\rangle_{C}|1\rangle_{D},
$$

where $\mid$ cat $\left._{ \pm}\right\rangle$denote positive and negative cat states $N_{ \pm}(|\alpha\rangle \pm|-\alpha\rangle)$ [14-16] in the CV mode $C$ and $\left\{|0\rangle_{D},|1\rangle_{D}\right\}$ is the Fock basis for DV mode $D$, with $N_{ \pm}=$ $1 / \sqrt{2 \pm 2 e^{-2 \alpha^{2}}}$ being the normalization factors and $K_{ \pm}$ adjustable weight parameters with $\left|K_{+}\right|^{2}+\left|K_{-}\right|^{2}=1$.

The CV part of the resource (1) is distributed to Alice, while the DV part goes to Bob. The source state to be teleported is an amplitude-matched CV qubit

$$
\left.\left.\mid \text { in }\rangle_{A}=X \mid \text { cat }_{+}\right\rangle_{A}+Y \mid \text { cat }_{-}\right\rangle_{A} .
$$

To implement the teleportation, Alice performs a joint measurement on this state and the CV part of the resource in the continuous-variable Bell basis defined by

$$
\begin{aligned}
\left|\Phi^{ \pm}\right\rangle_{A C} & =\frac{|\alpha\rangle_{A}|\alpha\rangle_{C} \pm|-\alpha\rangle_{A}|-\alpha\rangle_{C}}{\sqrt{2 \pm 2 e^{-4 \alpha^{2}}}} ; \\
\left|\Psi^{ \pm}\right\rangle_{A C} & =\frac{|\alpha\rangle_{A}|-\alpha\rangle_{C} \pm|-\alpha\rangle_{A}|\alpha\rangle_{C}}{\sqrt{2 \pm 2 e^{-4 \alpha^{2}}}} .
\end{aligned}
$$


The Bell measurement implemented in our work, discussed below, approximates a projection onto state $\left|\Phi^{-}\right\rangle$, which can be rewritten as

$$
\left|\Phi^{-}\right\rangle_{A C}=\frac{\left|\mathrm{cat}_{-}\right\rangle_{A}\left|\mathrm{cat}_{+}\right\rangle_{C}+\left|\mathrm{cat}_{+}\right\rangle_{A}\left|\mathrm{cat}_{-}\right\rangle_{C}}{\sqrt{2}} .
$$

In the event this state is detected in modes $A$ and $C$, the state of Bob's mode picks up the coefficients of the source $\mathrm{CV}$ qubit:

$$
\begin{aligned}
\mid \text { out }\rangle_{D} & \left.={ }_{A C}\left\langle\Phi^{-}\right|(\mid \text {in }\rangle_{A}|R\rangle_{C D}\right) \\
& =\frac{1}{\sqrt{2}}\left(X K_{-}|0\rangle_{D}+Y K_{+}|1\rangle_{D}\right),
\end{aligned}
$$

completing teleportation of the quantum information between continuous and discrete bases.

To perform the Bell state projection, we overlap modes $A$ and $C$ on a symmetric beam splitter, which transforms Bell states (3) into tensor products of a positive or negative cat state in one output mode and vacuum in the other:

$$
\begin{aligned}
\left|\Phi^{ \pm}\right\rangle_{A C} & \rightarrow \frac{(|\sqrt{2} \alpha\rangle \pm|-\sqrt{2} \alpha\rangle)_{A}|0\rangle_{C}}{\sqrt{2 \pm 2 e^{-4 \alpha^{2}}}}, \\
\left|\Psi^{ \pm}\right\rangle_{A C} & \rightarrow \frac{|0\rangle_{A}(|\sqrt{2} \alpha\rangle \pm|-\sqrt{2} \alpha\rangle)_{C}}{\sqrt{2 \pm 2 e^{-4 \alpha^{2}}}} .
\end{aligned}
$$

These four states can be distinguished by means of a parity measurement in modes $A$ and $C[13,17]$, which can be implemented using photon number discriminating detectors.

Limited by the current experimental technology of the resource state preparation [18], we work with moderate amplitudes $\alpha \sim 1 / 2$. In this regime, state $\left|\Phi^{-}\right\rangle$can be distinguished by simply projecting mode $A$ onto the singlephoton state. Indeed, a photon detection event cannot happen in response to states (6b). Furthermore, state $\left|\Phi^{+}\right\rangle$transforms into a positive cat state, whose Fock decomposition for a small $\alpha$ contains primarily the vacuum state. The negative cat state obtained from $\left|\Phi^{-}\right\rangle$, on the other hand, has the single-photon state as the leading term in its Fock decomposition and hence is much more likely to bring about a click (Supplemental Material [19]).

We tested the teleportation protocol in a simplified manner, emulating the input $\mathrm{CV}$ qubit by a coherent state of a varied phase in accordance with the relation

$$
\begin{aligned}
\mid \text { in }\rangle_{A} & =\left|\alpha e^{i \phi}\right\rangle_{A} \approx|0\rangle+\alpha|1\rangle e^{i \phi} \\
& \approx \frac{1+e^{i \phi}}{2}|\alpha\rangle+\frac{1-e^{i \phi}}{2}|-\alpha\rangle \\
& \approx \frac{1}{2}\left[\frac{\left|\mathrm{cat}_{+}\right\rangle_{A}}{N_{+}}+e^{i \phi} \frac{\left|\mathrm{cat}_{-}\right\rangle_{A}}{N_{-}}\right],
\end{aligned}
$$

which holds at $\alpha=0.5$ with an average fidelity of $95 \%$. For the input (7), the expected output state (5) then is

$$
\mid \text { out }\rangle_{D}=\frac{1}{2 \sqrt{2}}\left[\frac{K_{-}}{N_{+}}|0\rangle_{D}+e^{i \phi} \frac{K_{+}}{N_{-}}|1\rangle_{D}\right] \text {. }
$$

In this way, the protocol maps the phase of the input $\mathrm{CV}$ qubit onto a parallel of the DV qubit's Bloch sphere, with the latitude of that parallel being a function of $K_{ \pm}$. We choose to bring the output manifold to the equator by setting $K_{ \pm} \propto N_{\mp}$. This ensures that the weights of the vacuum and single-photon states in the output are equal, thereby allowing for a more precise characterization of the teleportation process.

Experiment.-We produce the hybrid resource state (1) following the method developed by Morin et al. [18] (Fig. 1). We employ two parametric down-conversion processes: one degenerate and the other nondegenerate, which take place in nonlinear crystals I and II. The crystals are periodically poled potassium titanyl phosphate and are pumped with frequency-doubled pulses at $390 \mathrm{~nm}$, generated by a Ti:sapphire laser with a repetition rate of $76 \mathrm{MHz}$ and a pulse width of $1.5 \mathrm{ps}$ [22].

The CV part of the resource is generated in crystal I, which initially produces a single-mode squeezed state with the squeezing parameter $\zeta=0.18$ [23]. This state approximates state $\mid$ cat $\left._{+}\right\rangle$with $\alpha_{+}=\sqrt{\zeta}[24,25]$. The DV part of the resource originates from crystal II, where a weak twomode squeezed state is created. The degree of squeezing in this state is much lower than that in the CV mode.

In order to entangle the two parts of the resource, the CV mode is "tapped" using a 5\% reflectivity beam splitter. The reflected mode is overlapped, on another beam splitter, with one of the modes emitted by crystal II and directed to a single-photon counting module $\mathrm{SPCM}_{1}$. The resource is obtained conditioned on a click of that detector.

If the photon that causes the click comes from crystal I, then it is subtracted from the single-mode squeezed state, producing a negative cat state in the CV mode $[14,15,26]$.

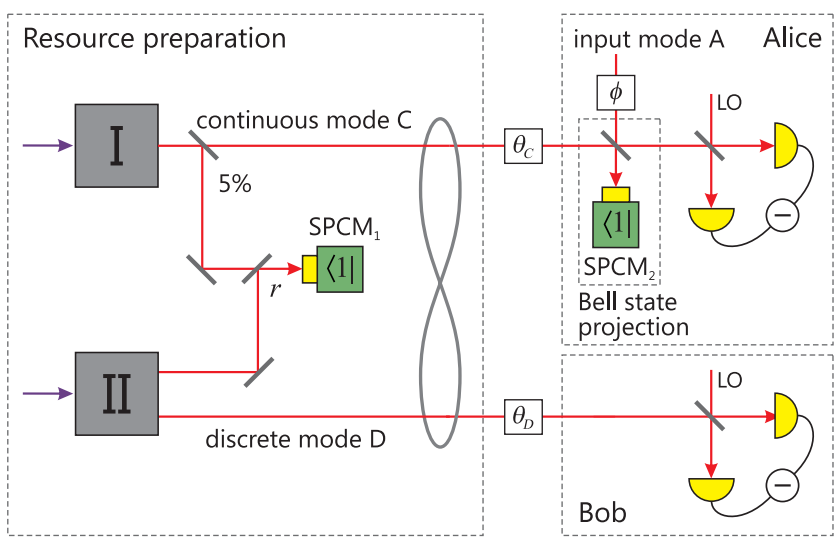

FIG. 1. Conceptual scheme of the experiment. The teleportation occurs from a CV qubit (a coherent state in our case) in input mode $A$ onto the DV vacuum-photon superposition in mode $D$. The preparation of the hybrid entangled resource (1) in modes $C$ and $D$ is heralded by a count of $\mathrm{SPCM}_{1}$, while the Bell state projection is conditioned on a click of $\mathrm{SPCM}_{2}$. The teleported state is measured by Bob using a homodyne detector. Another homodyne detector in mode $C$ serves the auxiliary purpose of the phase measurement. 
The DV mode is in the vacuum state in this case because of the low degree of two-mode squeezing obtained from crystal II. If, on the other hand, the photon comes from crystal II, it heralds the preparation of the single-photon state in the other output mode of that crystal [27]; the positive cat state in the $\mathrm{CV}$ mode is then undisturbed. Because the detector is fundamentally unable to distinguish between these options, their coherent superposition, given by Eq. (1), is produced.

The ratio of count rates $R_{\mathrm{IIII}}$ of $\mathrm{SPCM}_{1}$ from the two crystals determines the weight parameters $K_{ \pm}$of the resource (1). In order to comply with the condition $K_{ \pm} \propto N_{\mp}$, the rates should be related as

$$
\frac{R_{\mathrm{I}}}{R_{\mathrm{II}}}=\left(\frac{N_{+}}{N_{-}}\right)^{2}=\frac{1-e^{-2 \alpha_{-}^{2}}}{1+e^{-2 \alpha_{+}^{2}}} \approx \frac{1}{3} .
$$

Experimentally, this ratio is controlled by varying the reflectivity of the beam splitter in front of $\mathrm{SPCM}_{1}$. The total resource preparation rate is $R_{\mathrm{I}}+R_{\mathrm{II}} \approx 20 \mathrm{kHz}$.

We characterize the resource state by means of two-mode homodyne tomography. To that end, we temporarily remove the beam splitter associated with the Bell measurement and conduct a series of field quadrature measurements in modes $C$ and $D$. The optical phases $\theta_{C, D}$ of the state in the two modes, required for the state reconstruction, are obtained from the quadrature data statistics. The data in the continuous channel, acquired without conditioning on an event in $\mathrm{SPCM}_{1}$, correspond to a squeezed vacuum state, so the quadrature variance is related to the phase according to $\left\langle X_{C}^{2}\right\rangle \sim \cos 2 \theta_{C}$, allowing us to extract $\theta_{C}$. Phase $\theta_{D}$ is determined using correlation $\left\langle X_{C} X_{D}\right\rangle \propto \cos \left(\theta_{D}-\theta_{C}\right)$, which is characteristic of state (1) as demonstrated in Supplemental Material [19].

The resource state is reconstructed using the maximumlikelihood algorithm $[28,29]$, correcting for a $54 \%$ detection efficiency (see the efficiency budget in Supplemental Material [19]). The projection of the reconstructed state onto $|1\rangle_{D}$ in the discrete mode is a positive cat state of amplitude $\alpha_{+}=0.44$ [Fig. 2(a)]. Projection onto $|0\rangle_{D}$ [Fig. 2(b)] corresponds to the photon-subtracted positive cat state, which is a negative cat state of amplitude $\alpha_{-}=0.74$, in approximate agreement with theoretical expectation $\alpha_{-}=\sqrt{3} \alpha_{+}$(Supplemental Material [19]). The overall reconstructed resource state has $96 \%$ fidelity to state (1) with $\alpha=0.56$.

The mismatch between $\alpha_{+}$and $\alpha_{-}$is an inherent shortcoming of the resource preparation protocol of Ref. [18]. An alternative method [30] does not have this drawback but imposes much stronger limitations on the amplitude of the $\mathrm{CV}$ resource mode.

We perform the Bell projection on behalf of Alice by overlapping the input coherent state with the CV mode of the resource on a symmetric beam splitter and subjecting one of the resulting modes to the nondiscriminating singlephoton detection via $\mathrm{SPCM}_{2}$. A narrow band spectral filter in front of the detector, combined with the spatial filtering by means of a single-mode optical fiber, ensures nearly
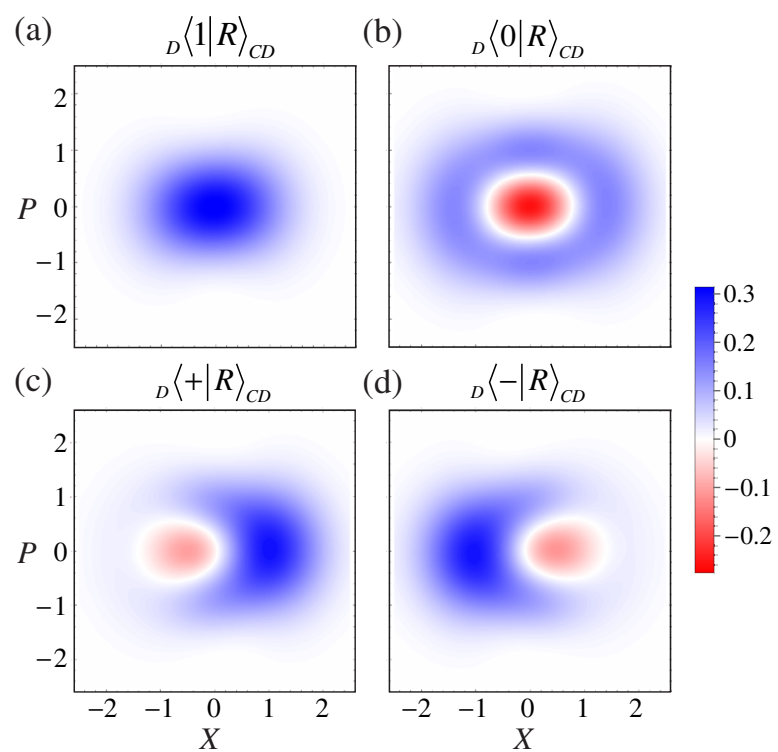

FIG. 2. Wigner functions of the CV mode of the entangled resource state, reconstructed with a $54 \%$ efficiency correction, projected in the DV mode onto various qubit states: (a) $|1\rangle_{D}$; (b) $|0\rangle_{D}$; (c) $|+\rangle=\left(|0\rangle_{D}+|1\rangle_{D}\right) / \sqrt{2}$; (d) $|-\rangle=\left(|0\rangle_{D}-|1\rangle_{D}\right) / \sqrt{2}$.

perfect indistinguishability of modes $A$ and $C$ when they reach the detector. For the $\mathrm{CV}$ amplitude used in our work, state $\left|\Phi^{-}\right\rangle$brings about a count event with the probability that is about 9 times higher than the three other Bell states taken together (Supplemental Material [19]). Therefore, such a count, in coincidence with a click from $\mathrm{SPCM}_{1}$, heralds a successful event of the teleportation protocol. Such events occur in our experiment at a rate of $350 \mathrm{~s}^{-1}$, which corresponds to the probability of successful teleportation of $1 / 57$. The data collected in our experiment correspond to a total of 524288 events.

To verify the teleportation and measure its fidelity, we subject the teleported state in mode $D$ to optical homodyne tomography. The output state reconstruction and its comparison with the input state requires the phase $\phi, \theta_{C}, \theta_{D}$ to be known at each moment in time. The values of $\theta_{C}$ and $\theta_{D}$ are evaluated from the resource state as discussed above, while the input CV qubit phase $\phi$ is obtained from the average reading of Alice's homodyne detector: $\left\langle X_{C}\right\rangle \propto \cos \phi$. This phase, as well as the phase $\theta_{D}$ of the discrete resource mode, is varied by means of the piezoelectric transducer. In this way, for each value of $\phi$, a tomographically complete set of quadrature measurements is acquired.

Results.-Figure 3(a) shows the density matrices of the teleported DV qubit states for a set of the input CV qubit phases $\phi$. The data are corrected for the $54 \%$ detection efficiency on Bob's side.

The off-diagonal elements are complex numbers whose argument is approximately equal to $\phi$ [Fig. 3(b)], as expected from Eq. (8). The standard deviation between the phases of the input and teleported states is $0.067 \mathrm{rad}$ or $3.8^{\circ}$. This can be compared with the classical measure-andprepare strategy, which allows reproducing the phase of a 

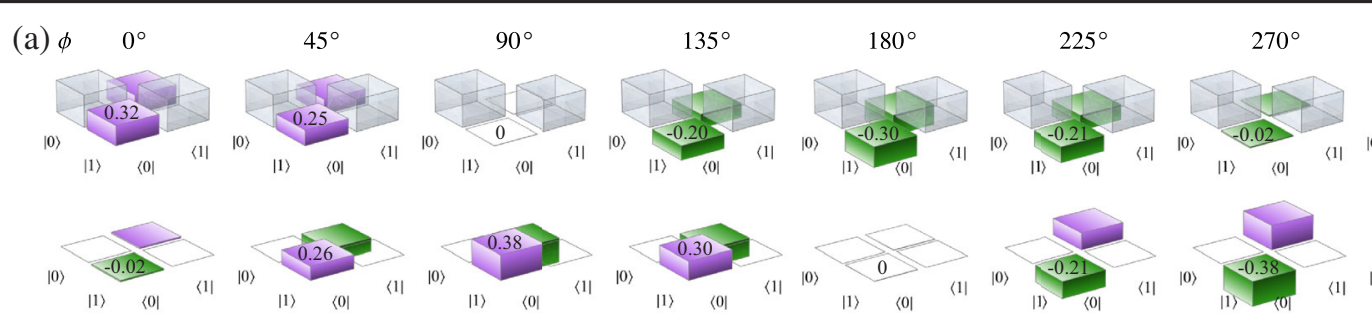

$315^{\circ}$
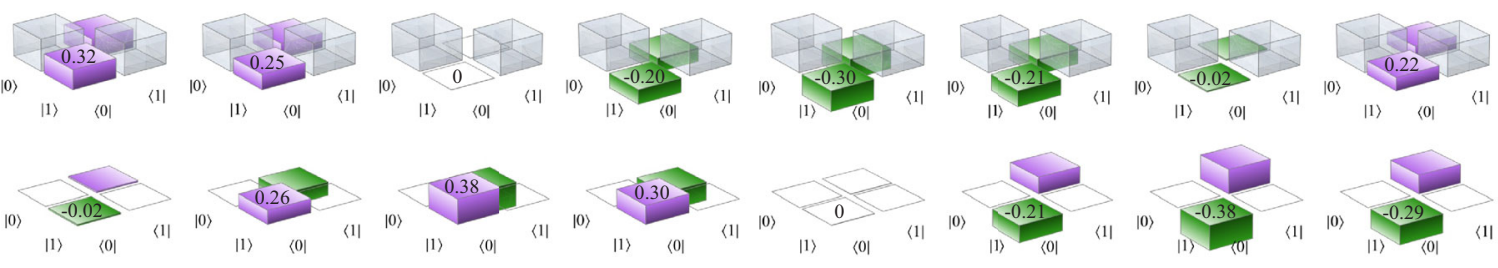

(b)

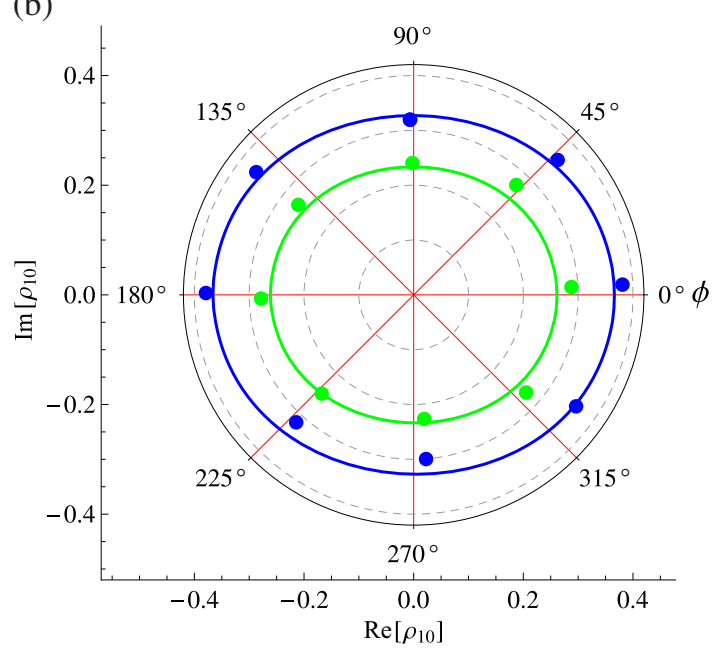

(c)
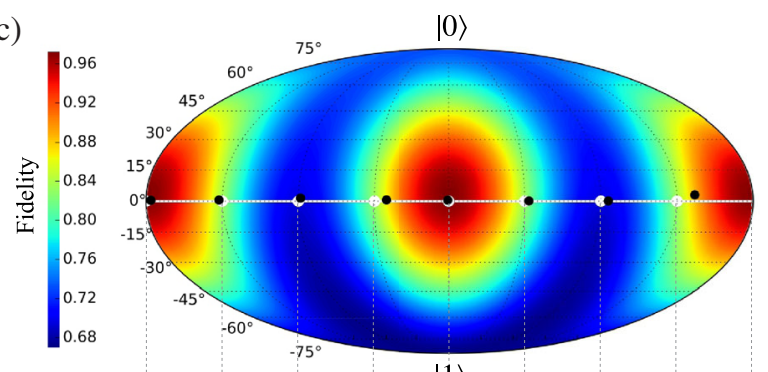

|1〉

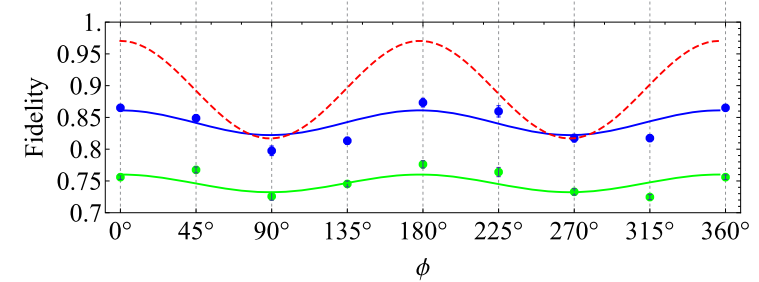

FIG. 3. Experimental results. (a) Density matrices of the teleported state, as measured by Bob's homodyne detector, for different phases of the input qubit, reconstructed with a correction for $54 \%$ of detection efficiency. Top row: Real part. Bottom row: Imaginary part. The coloring scheme distinguishes the diagonal (gray) as well as positive (purple) and negative (green) off-diagonal matrix elements for viewing convenience. (b) Off-diagonal elements of the teleported qubit's density matrices, dots. Lines: Simulation results. Blue (green) data: With (without) efficiency correction. The radial lines indicate the corresponding input state phases. (c) Color map: Expected fidelity of the protocol, obtained via a numeric simulation, over the full Bloch sphere. Dots show the states tested in the experiment. White: Target. Black: Measured. (d) Fidelity between the states reconstructed from the experimental data with an efficiency correction and theoretical target states. Error bars correspond to one standard deviation. The solid lines are theoretical predictions taking into account all experimental imperfections including the phase estimation uncertainty. The red dashed line ignores the phase uncertainty.

coherent state with standard error $1 /(\sqrt{2} \alpha) \approx 1.41$, over 20 times larger than the phase error in our experiment.

The magnitude of the off-diagonal elements varies periodically a function of $\phi$, directly affecting the teleportation fidelity [Figs. 3(b) and 3(d)]. This effect is due to the simplified realization of the Bell measurement and approximating the input $\mathrm{CV}$ qubit by a coherent state. For $\theta_{C} \approx 0$, as is the case in our experiment, both these factors reduce the fidelity most significantly at $\phi= \pm \pi / 2$ and are minimized at $\phi=0, \pi$.

The teleportation fidelity is further influenced by phase uncertainties. First, because we do not actively stabilize $\theta_{C}$, it fluctuates with a mean deviation of $0.53 \mathrm{rad}$. Second, there exists an $\sim 0.5$ rad statistical uncertainty of evaluating the resource phase difference $\theta_{D}-\theta_{C}$. This is because the acquisition of the correlated quadrature data required for this evaluation is conditioned on clicks in $\mathrm{SPCM}_{1}$, which occur at a relatively low rate of $20 \mathrm{kHz}$. The combined effect of the phase uncertainties is to smoothen the fidelity variation as a function of $\phi$ and degrade the average fidelity by about $5 \%$, as can be seen by comparing the red and blue lines in Fig. 3(d).
Based on the result of our measurement, we have calculated the expected fidelity of our protocol for an arbitrary input CV qubit [Fig. 3(c)]. The model includes the same resource state and simplified realization of the Bell measurement as in our experiment but assumes no phase measurement uncertainties. Averaging over the Bloch sphere gives the mean fidelity of $79.5 \%$, which exceeds the classical teleportation benchmark $2 / 3$ [31]. Surprisingly, nonidealities of the resource state, discussed above, do not lead to a significant drop of the fidelity: With our resource and ideal Bell projection, the average fidelity would be $98 \%$. The positions of the experimental teleportation output states on the Bloch sphere are shown by black dots. As intended, the obtained states are close to the equator, marked by the white line.

Summary and outlook.-We believe our scheme to be the first application of the discrete-continuous hybrid entanglement $[18,30]$ in quantum information processing. Intended as a conceptual proof-of-principle experiment, our work involves a number of approximations or compromises. Some of these are of a technical nature and can be rectified using existing technology. For example, while our 
Bell measurement can isolate only one of the CV Bell states, efficient number-resolving detectors [32] would enable distinguishing all four of them deterministically through a photon number parity measurement. Also, we tested our protocol only with qubits on the equator of the Bloch sphere. In order to apply it to bona fide CV qubits [6], such qubits can be prepared using the technique of Ref. [33]. Its integration with our experiment is straightforward but requires the significant enhancement of all nonlinear optical processes involved in order to bring up the photon count event rate.

Other limitations are associated with the entangled CV-DV resource whose relatively low amplitude, which is furthermore unequal for the positive and negative cat components, is inherent to the preparation method [18]. Finding a technique for preparing a CV-DV resource that is free from these shortcomings is an important subject for future investigations [34].

We thank $\mathrm{H}$. Jeong for inspiring discussions. We acknowledge financial support from Ministry of Education and Science of the Russian Federation (Agreement No. 14.582.21.0009, ID RFMEFI58215X0009). A. L. is supported by NSERC and by a CIFAR Fellowship.

[1] G. Kurizki, P. Bertet, Y. Kubo, K. Mølmer, D. Petrosyan, P. Rabl, and J. Schmiedmayer, Quantum technologies with hybrid systems, Proc. Natl. Acad. Sci. U.S.A. 112, 3866 (2015).

[2] S. L. Braunstein and P. van Loock, Quantum information with continuous variables, Rev. Mod. Phys. 77, 513 (2005).

[3] P. Kok, W. J. Munro, K. Nemoto, T. C. Ralph, J. P. Dowling, and G. J. Milburn, Linear optical quantum computing with photonic qubits, Rev. Mod. Phys. 79, 135 (2007).

[4] U. L. Andersen, J. S. Neergaard-Nielsen, P. van Loock, and A. Furusawa, Hybrid discrete- and continuous-variable quantum information, Nat. Phys. 11, 713 (2015).

[5] G. Qi, C. Liu-Yong, W. Hong-Fu, and Z. Sho, Universal quantum computation using all-optical hybrid encoding, Chin. Phys. B 24, 040303 (2015).

[6] S.-W. Lee and H. Jeong, Near-deterministic quantum teleportation and resource- efficient quantum computation using linear optics and hybrid qubits, Phys. Rev. A 87, 022326 (2013).

[7] A. E. Ulanov, I. A. Fedorov, A. A. Pushkina, Y. Kurochkin, T. C. Ralph, and A. I. Lvovsky, Undoing the effect of loss on quantum entanglement, Nat. Photonics 9, 764 (2015).

[8] A. E. Ulanov, I. A. Fedorov, D. Sychev, P. Grangier, and A. I. Lvovsky, Loss-tolerant quantum enhanced metrologyand state engineering via the reverse Hong-Ou-Mandel effect Nat. Commun. 7, 11925 (2016).

[9] S. Pirandola, J. Eisert, C. Weedbrook, A. Furusawa, and S. L. Braunstein, Advances in quantum teleportation, Nat. Photonics 9, 641 (2015).

[10] S. Pirandola and S. L. Braunstein, Physics: Unite to build a quantum Internet, Nature (London) 532, 169 (2016).
[11] C. H. Bennett, G. Brassard, C. Crépeau, R. Jozsa, A. Peres, and W. K. Wootters, Teleporting an Unknown Quantum State via Dual Classical and Einstein-Podolsky-Rosen Channels, Phys. Rev. Lett. 70, 1895 (1993).

[12] S. Takeda, T. Mizuta, M. Fuwa, P. van Loock, and A. Furusawa, Deterministic quantum teleportation of photonic quantum bits by a hybrid technique, Nature (London) 500, 315 (2013).

[13] K. Park, S. W. Lee, and H. Jeong, Quantum teleportation between particlelike and fieldlike qubits using hybrid entanglement under decoherence effects, Phys. Rev. A 86, 062301 (2012).

[14] A. Ourjoumtsev, R. Tualle-Brouri, J. Laurat, and P. Grangier, Generating optical Schrödinger kittens for quantum information processing, Science 312, 83 (2006).

[15] J. S. Neergaard-Nielsen, B. M. Nielsen, C. Hettich, K. Mlmer, and E.S. Polzik, Generation of a Superposition of Odd Photon Number States for Quantum Information Networks, Phys. Rev. Lett. 97, 083604 (2006).

[16] K. Wakui, H. Takahashi, A. Furusawa, and M. Sasaki, Photon subtracted squeezed states generated with periodically poled KTiOPO4, Opt. Express 15, 3568 (2007).

[17] H. Jeong, M.S. Kim, and J. Lee, Quantum-information processing for a coherent superposition state via a mixed entangled coherent channel, Phys. Rev. A 64, 052308 (2001).

[18] O. Morin, K. Huang, J. Liu, H. Le Jeannic, C. Fabre, and J. Laurat, Remote creation of hybrid entanglement between particle-like and wave-like optical qubits, Nat. Photonics 8 , 570 (2014).

[19] See Supplemental Material at http://link.aps.org/ supplemental/10.1103/PhysRevLett.118.160501 for additional theoretical analysis and discussion of inefficiency sources, which includes Refs. [20,21].

[20] T. Aichele, A. I. Lvovsky, and S. Schiller, Optical mode characterization of single photons prepared by means of conditional measurements on a biphoton state, Eur. Phys. J. D 18, 237245 (2002).

[21] R. Kumar, E. Barrios, A. MacRaea, E. Cairns, E. H. Huntington, and A. I. Lvovsky, Versatile wideband balanced detector for quantum optical homodyne tomography, Opt. Commun. 285, 5259 (2012).

[22] S. R. Huisman, N. Jain, S. A. Babichev, F. Vewinger, A.-N. Zhang, S.-H. Youn, and A. I. Lvovsky, Instant single-photon Fock state tomography, Opt. Lett. 34, 2739 (2009).

[23] Loss-free squeezing with parameter $\zeta$ reduces the squeezed quadrature variance by a factor of $e^{-2 \zeta}$ with respect to the vacuum noise level.

[24] A. Ourjoumtsev, F. Ferreyrol, R. Tualle-Brouri, and P. Grangier, Preparation of non-local superpositions of quasi-classical light states, Nat. Phys. 5, 189 (2009).

[25] A. I. Lvovsky, in Photonics Volume 1: Fundamentals of Photonics and Physics, edited by D. Andrews (Wiley, West Sussex, United Kingdom, 2015), pp. 121-164.

[26] J. S. Neergard-Nielsen, M. Takeuchi, K. Wakui, H. Takahashi, K. Hayasaka, M. Tateoka, and M. Sasaki, Photon subtraction from traveling fields-Recent experimental demonstrations, Progr. Informat. 8, 5 (2011).

[27] A. I. Lvovsky, H. Hansen, T. Aichele, O. Benson, J. Mlynek, and S. Schiller, Quantum State Reconstruction of the SinglePhoton Fock State, Phys. Rev. Lett. 87, 050402 (2001). 
[28] A. I. Lvovsky, Iterative maximum-likelihood reconstruction in quantum homodyne tomography, J. Opt. B 6, S556 (2004).

[29] J. Řeháček, Z. Hradil, E. Knill, and A. I. Lvovsky, Diluted maximum-likelihood algorithm for quantum tomography, Phys. Rev. A 75, 042108 (2007).

[30] H. Jeong, A. Zavatta, M. Kang, S. Lee, L. S. Costanzo, S. Grandi, and M. Bellini, Generation of hybrid entanglement of light, Nat. Photonics 8, 564 (2014).

[31] S. Oh, S. Lee, and H. Lee, Fidelity of quantum teleportation through noisy channels, Phys. Rev. A 66, 022316 (2002).
[32] F. Marsili et al., Detecting single infrared photons with $93 \%$ system efficiency, Nat. Photonics 7, 210 (2013).

[33] J. S. Neergaard-Nielsen, M. Takeuchi, K. Wakui, H. Takahashi, K. Hayasaka, M. Takeoka, and M. Sasaki, Optical Continuous-Variable Qubit, Phys. Rev. Lett. 105, 053602 (2010).

[34] D. Sychev, A. E. Ulanov, A. A. Pushkina, M. W. Richards, and A. I. Lvovsky, Breeding the optical Schrödinger's cat state, arXiv:1609.08425. 